\title{
Assisted reach and transfers in individuals with tetraplegia: towards a solution
}

\author{
GT Allison and KP Singer \\ School of Physiotherapy, Curtin University, Selby Street, Shenton Park 6014, Western Australia
}

\begin{abstract}
The purpose of this study was to investigate the influence of a prototype trunk orthosis to assist an individual with tetraplegia. A single case study (26 year old male, C5 motor complete) using an interrupted time series analysis was conducted to investigate the individual's ability to reach, forward and laterally, and transfer with and without the orthosis. All tasks were performed on an AMTI ${ }^{\circledR}$ force platform in the long sitting position, with landmarks of the trunk and limbs recorded using Peak Performances Technologies ${ }^{\circledR}$ motion analysis system. After a familiarisation period ten trials were attempted for each phase of the analysis. With the orthosis the subject altered the sitting posture and significantly $(\mathrm{F}=9.55, P=0.003)$ increased the distance the subject was able to reach. The median frequency of the centre of pressure (COP) displacement during the reaching task was not significantly altered. The ability to displace the COP when attempting to transfer increased from $16.0( \pm 3.4 \mathrm{~cm})$ to $19.6( \pm 2.5 \mathrm{~cm})$, however this was not statistically significant. The likely user population, the overall functional benefits, the compliance of the users and possible modifications to the device to facilitate use with functional electrical stimulation are all possible directions for future research.
\end{abstract}

Keywords: orthosis; spinal cord injury; rehabilitation; function; tetraplegia

\section{Introduction}

The application of biomechanical analysis to human movement for both normal and abnormal conditions has led to the development of fundamental motion analysis paradigms that are the basis for many rehabilitation intervention regimens. Within the spinal cord injury (SCI) population, this is illustrated by the development of orthotic and/or functional electrical stimulation (FES) interventions which have been based on the motion analysis of normal human gait. ${ }^{1,2}$ To date the effects of the application of an orthotic device based on the biomechanical analysis of the lateral transfer are yet to be reported in the literature.

The ability to transfer in individuals with tetraplegia represents a significant factor in overall functional mobility and independence. Approximately 55\% of all individuals with SCI have tetraplegia. About half of them have weak triceps and the majority of these individuals can not transfer independently. The variance in the performance is related to factors other than neurological lesion level. ${ }^{3}$ The ability to transfer therefore represents a major clinical rehabilitation goal, yet has received little biomechanical inspection.

Correspondence: Dr Garry Allison, BEd (Hon), BAppSci (Hon) MEd, Ph.D., School of Physiotherapy, Curtin University, Selby Street, Shenton Park 6014 or 6008, Western Australia.
Many biomechanical factors have been anecdotally associated with the ability to transfer, for example, arm length (in absolute terms and relative to the height of the shoulders above the floor and the length of the torso), muscle strength and hand placement. Bergstrom and co-workers ${ }^{4}$ demonstrated objectively a significant difference in fat mass, percentage body fat and the placement of the hand relative to the acromion process when attempting to lift between individuals with C6 tetraplegia who could and could not transfer. There was no difference between the groups for functional arm length, sitting height, age or body mass.

Findings from our research group, have previously demonstrated that there are two movement strategies adopted by individuals with SCI when transferring. ${ }^{5}$ It was found that some individuals performed the lateral transfer using a rotatory movement pattern, where the head moved in the opposite direction to the pelvis. Others performed a translational strategy where the head and pelvis moved in concert. The fact that there are at least two movement strategies may account for the variability in the factors associated with the ability to transfer reported in the literature. It was also demonstrated that individuals with weak triceps attempted to transfer with a rotatory pattern rather than a translatory pattern. ${ }^{5}$

Differences between the two movement strategies may also exist in the phasic activity of the elbow 
extensors and latissimus dorsi during the transfer. ${ }^{6}$ There may also be a difference in the pre-transfer posture of the individual. The rotatory pattern has a more upright pre-transfer body position, with higher acromion processes and wrists placed closer to the body when compared to the translatory group. ${ }^{5}$ Unpublished data from our laboratory also indicates that individuals with tetraplegia are less stable (higher mean frequency of movement) in the pre-transfer position when compared to paraplegics.

It has been hypothesised that the poor trunk stability of the individual with a mid- to high level of tetraplegia may limit upper extremity function. ${ }^{7}$ This may include upper extremity reaching performance and possibly the ability to transfer. The effect of increased trunk support on upper limb reaching function in individuals with tetraplegia has yet to be reported.

In recognition of the functional significance of upper limb reaching and the ability to transfer in individuals with tetraplegia, and based on the above biomechanical analyses of the long sitting transfer, a prototype orthosis was developed. A study was then undertaken to investigate the biomechanical and functional adaptations during reaching and transfer tasks in an individual with a C5 level tetraplegia when using a prototype trunk orthosis.

\section{Methods}

\section{Subject}

The case study involved a 26 year old male $(56.6 \mathrm{~kg}$, $188 \mathrm{~cm}$ lying) who had received a cervical spine injury following a diving accident 5.8 years previously. He presented with a motor complete C5 tetraplegia (with some sensory sparing distal to the motor lesion Frankel B). ${ }^{8}$ At the time of testing he was studying mechanical drafting approximately $4 \mathrm{~h}$ a week. He lives independently with $33 \mathrm{~h}$ attendant care per week and family support at other times. He had previously received posterior deltoid muscle transfer surgery on his left arm to assist in elbow extension. He could fully extend both elbows with gravity eliminated, against gravity the range was reduced approximately 15 degrees.

$\mathrm{He}$ reports that he is independent in bed activities when he can use a rope ladder. He performs some level transfers with an assistant positioning his lower limbs and assisting with the use of a sliding board. He is dependent in all other transfers. He is able to perform basic mobility wheelchair tasks and is able to perform some body repositioning tasks with limited success thereby necessitating some assistance.

The subject reported that at the time of testing he had no pain in the shoulders or arms and that he did not have pain when attempting to transfer.

$\mathrm{He}$ received and signed an informed consent form and ethical approval was obtained from the Curtin University Human Research Ethics Committee.

\section{Orthotic design}

The concept of an orthosis and the biomechanical analyses of the long sitting transfer were presented to a bio-engineer and orthotist. The design prototype was constructed with three design requirements: (i) to stabilise the trunk, thus making the torso more rigid and therefore act more like a single segment rather than a series of independent segments. (ii) to provide increased amplitude of movement over the base of suport by allowing the upper body to move to positions that may be held without the use of the upper limbs for support, and (iii) to allow voluntary movement into new ranges, in particular into forward flexion, during the transfer attempts.

The prototype design incorporated a rigid frame and with an adjustable hinge, lumbar support, anterior chest plate and thigh supports (see Figure 1). All adjustable straps were made from Velcro ${ }^{\mathbb{R}}$.

\section{Testing protocol}

Three tasks (two reaching tasks and one transfer task) were performed on an $\mathrm{AMTI}^{\circledR}$ force platform in the long sitting position under two conditions: with and without an orthosis. After a familiarisation period of (approximately 10 trials) ten trials were attempted for each phase of the analysis.

The first task was a maximal forward reach using two hands held for $1.5 \mathrm{~s}$. The second was a lateral

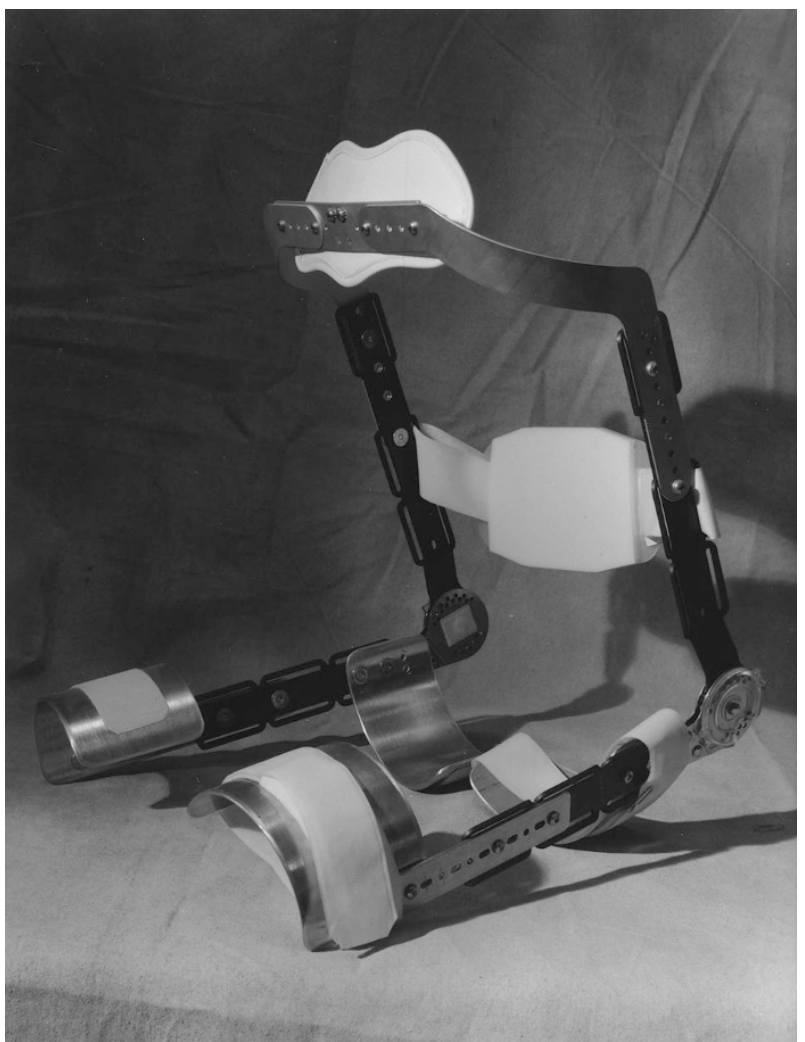

Figure 1 Antero-lateral view of the orthosis 


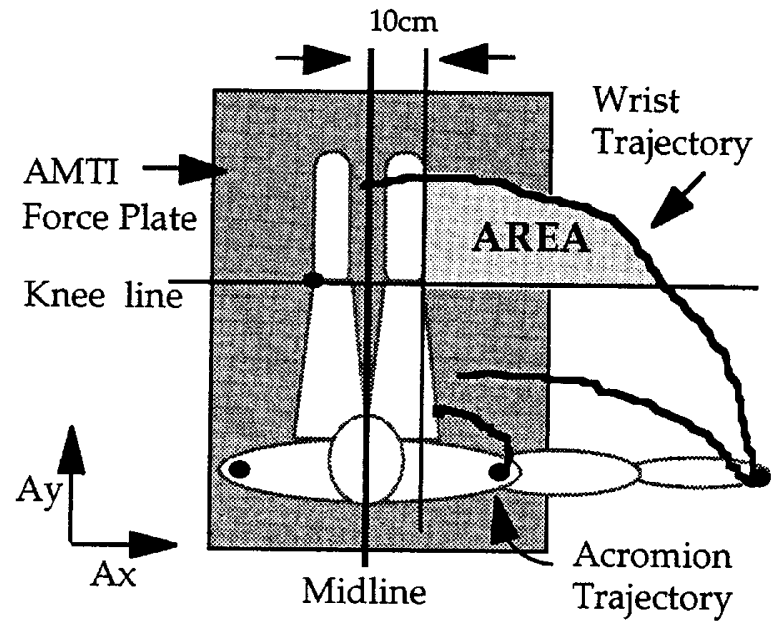

Figure 2 Overhead schematic diagram of the subject position for the reaching tasks. Area of reach is defined by the wrist trajectory, the knee line and a line $10 \mathrm{~cm}$ from the mid-line

reaching task. During the second task the subject reached as far as possible laterally, held the position for $1.5 \mathrm{~s}$, then reached forward by circumscribing the largest arc possible whilst maintaining the upper extremity extended. The trajectory of the wrists relative to the knees and body mid-line as viewed from an overhead video camera was recorded. The area of reach (see Figure 2) was quantified by calculating the area between the wrist trajectory, the knee line and a parallel line $10 \mathrm{~cm}$ from the mid-line.

During the static hold, stability assessments were made from the displacement of the centre of pressure (COP) which was recorded at $100 \mathrm{~Hz}$ on a PC via Peak Performances Technologies ${ }^{\circledR}$ software. The raw data were transferred to an Excel $^{\mathbb{R}}$ database, where the COP co-ordinates (Ax and Ay) were calcualted. The median frequency of the displacement of the COP was determined from the power density spectrum calculated from a Fast Fourier Transformation (FFT) ${ }^{9}$ A minimum of 128 data points were used for each static hold phase.

During the lateral reaching task the dynamic stability of the individual was assessed by determining the amplitude of the excursion of the COP laterally.

The third task was a long sitting lateral movement on the same surface (force plate). This movement pattern was believed to mimic the motor strategy of an individual attempting to perform a lateral transfer. Lateral and posterior views of the spatial models from reflective landmarks were recorded using two video cameras (see Figure 3). The Peak Performances Technologies ${ }^{\circledR}$ motion analysis system were used to calculate 2-D Cartesian co-ordinates of all the landmarks. From these co-ordinates the pre-transfer postures and lateral displacement of the pelvis were determined for each trial. Movement analysis crosscorrelations between the lateral displacements of the

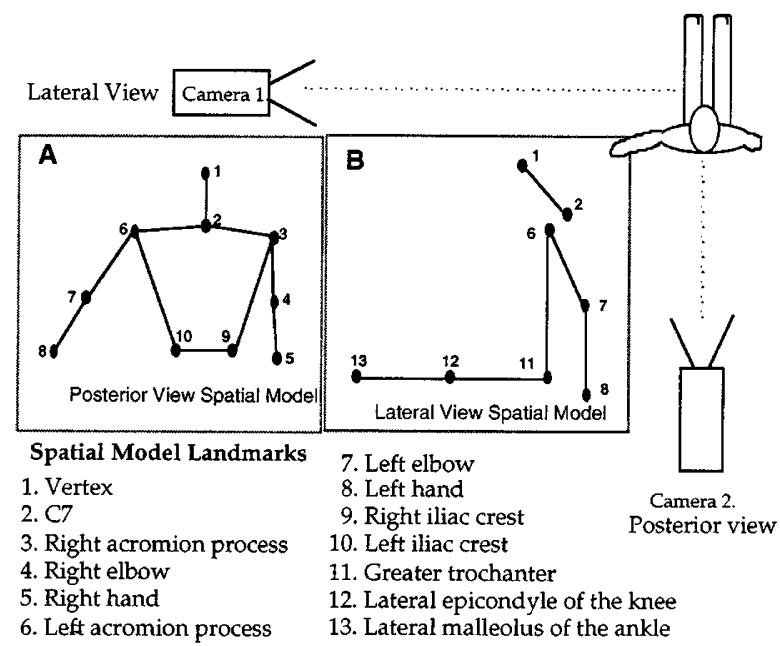

Figure 3 Schematic diagram of landmarks and camera position for the lateral and posterior view spatial models used for the motion analysis

head and the pelvis were used to identify the relative synchronisation of their displacements during the dynamic phase of the transfer. These were used to quantify the movement strategy (rotatory or translatory) as previously reported by this research group. ${ }^{5}$ The force plate trigger was synchronised with the video by a frame marker.

\section{Statistical procedures}

Repeated assessments of one individual violates the parametric statistical assumption of independence. ${ }^{10}$ Therefore interrupted time-series analysis (ITSA) was used to determine the effect of the intervention (orthosis) by controlling for autocorrelation of scores. It was recognised however that with less than 25 observations in each phase the power of the statistical process was decreased thereby increasing the chances of a type II error. ${ }^{11}$ Statistical significance was accepted at the 0.05 level of confidence.

\section{Results}

\section{Subjective findings}

The subject requested standby assistance when performing all tasks when without the orthosis. Assistance was required on two occasions when the subject failed to maintain his balance.

When the orthosis was fitted no standby assistance was required. The subject reported that the orthosis was 'too tall' for him and the chest plate pressed against the lower borders of his clavicles. During one attempted transfer he was able to lift himself briefly, from the supporting surface, a task he had not been able to perform since his injury. The subject stated that in assessing the benefit of the orthosis it was 
necessary to balance the improved function with the inconvenience of putting the orthosis on and taking it off. This issue needs further investigation.

\section{Posture}

Figure 4 illustrates the lateral profile of the subject with and without the orthosis in the pre-transfer posture. With the orthosis the subject was able to flex further forward and there was increased extension of the lumbar spine. This altered posture is reflected by significant increases in the relative position of the seventh cervical spinal process relative to the knee line during the transfer $\left(\mathrm{F}=10.01, \mathrm{df}_{2,18} P=0.002\right)$.

\section{Reaching}

The mean area of reach past the line of the knees was significantly increased $\left(\mathrm{F}=9.55 \mathrm{df}_{2,12} P=0.003\right)$ from $285 \pm 80 \mathrm{~cm}^{2}$ to $1770 \pm 308 \mathrm{~cm}^{2}(620 \%$ increase). These increases were a result of the change in trunk posture rather than improved upper extremity extension. The wrist trajectories drawn relative to the knee line and relative to the acromion process (see Figure 6) clearly illustrate this.

The median frequency of the COP during the static hold reaching tasks was not statistically different between conditions. With the orthosis, the median

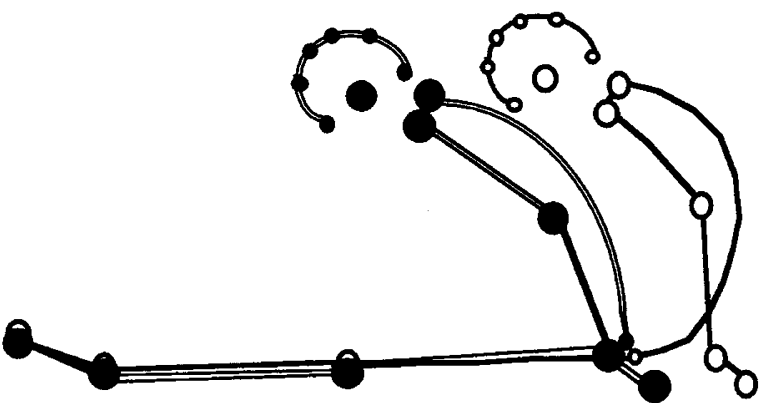

Figure 4 Lateral profile of the pre-transfer posture with and without $(\bigcirc)$ the orthosis

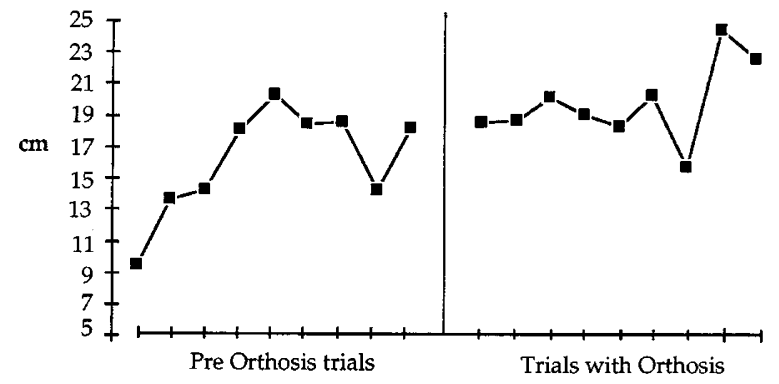

Figure 5 Graphical representation of lateral displacement of the pelvis during an attempted lateral transfer with and without the orthosis frequencies ranged between 0.4 and $6.9 \mathrm{~Hz}$ (mean $2.0 \mathrm{~Hz}, \mathrm{SD} 2.3 \mathrm{~Hz}$ ) and without the orthosis 0.5 and $11.6 \mathrm{~Hz}$ (mean $2.9 \mathrm{~Hz}$, SD $3.4 \mathrm{~Hz}$ ).

During the lateral reaching task, the mean lateral displacement of the COP was significantly greater with the orthosis $(2.6 \mathrm{~cm} \mathrm{SD} 0.87 \mathrm{~cm})$ than without the orthosis $(1.0 \mathrm{~cm} \mathrm{SD} 0.23 \mathrm{~cm})$.

\section{Transferring}

The subject demonstrated a rotatory movement strategy independent of the use of the orthosis since all trials had strong negative correlations between the lateral displacement of the head and pelvis. The mean (and standard deviation) correlation coefficient over the ten trials without the orthosis was -0.98 (SD 0.02) and with the orthosis -0.84 (SD 0.07). The coefficient of variation for each phase was $2.0 \%$ and $8.3 \%$ respectively.

The lateral displacement of the pelvis for the tenth trial for each testing protocol was lost due to data transformation difficulties. Figure 5 illustrates the time series data of nine repeated trials for the lateral displacement of pelvis during the transfer with and without the orthosis. The mean distance the pelvis was

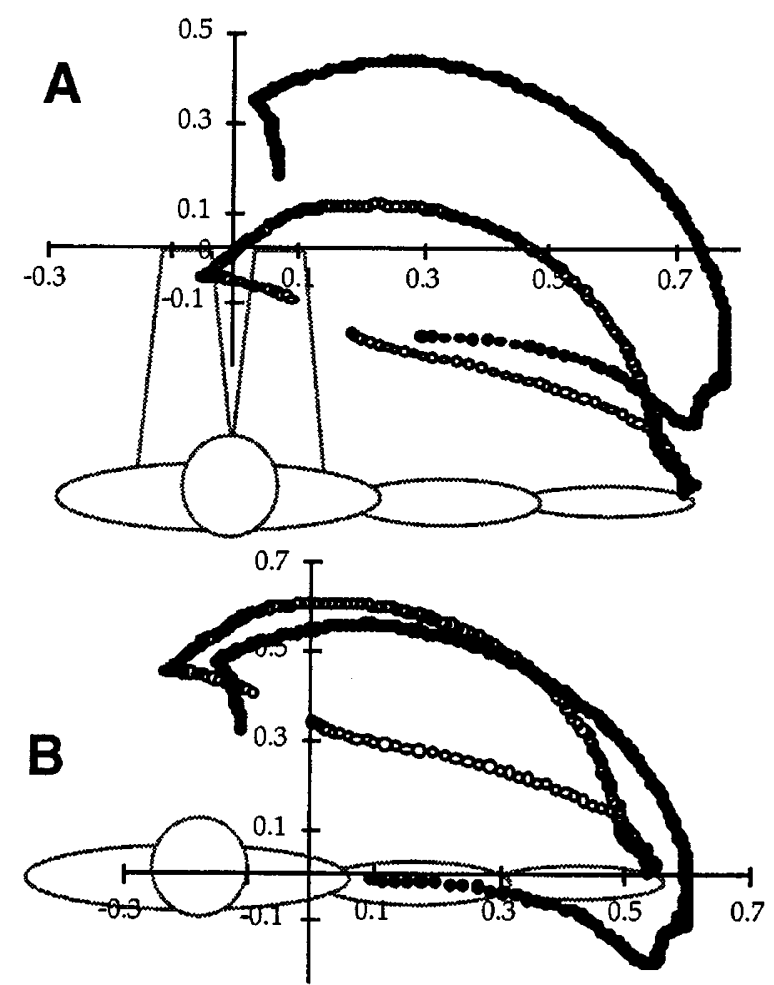

Figure 6 Overhead view of wrist trajectories of lateral reach task with (O) and without $(\bigcirc)$ the orthosis. (A) Illustrates the absolute difference between reaching with and without the orthosis relative to the knee line. (B) Illustrates the wrist trajectories relative to the acromion process. Body image is position without orthosis 
transferred laterally without the brace was 16.0 $( \pm 3.4 \mathrm{~cm})$ and with the brace $19.6( \pm 2.5 \mathrm{~cm})$. This was not statistically significant using an ITSA.

\section{Discussion}

The fact that the subject lifted the pelvis clear of the supporting surface shows that the orthosis did alter some biomechanical factors which improved his ability to lift. Bergstrom and co-workers ${ }^{4}$ used the ability to lift the pelvis as a requirement for independent transfer. If one uses this classification, the orthosis improved the performance of this subject. However, the likelihood of the subject using the orthosis in the present configuration was limited therefore future use of the system requires significant design modifications. This is supported by the feedback of the subject. This would incorporate positioning the vertical arms anteriorly and more medially on the chest. Furthermore, the utility donning and doffing the orthosis needs to be balanced with the increases in function. These issues may be considered in an overall quality of life assessment procedure.

\section{Pre-task postures}

The orthosis altered the long sitting posture of the subject by allowing him to sit forward with a more extended lumbar and lower thoracic spine. Without the orthosis the subject tended to sit back onto his sacrum with his pelvis rotated posteriorly. With the orthosis the pelvis was rotated anteriorly and the subject tended to sit more on his ischial tuberosities. This alteration in posture is directly reflected in the increased reaching capability when assessed by the area circumscribed in front of the knee-line.

Similarly, in the pre-transfer posture the subject was flexed further forward. It has been hypothesised that individuals with long arms relative to their stem (torso) length are more likely to transfer independently than individuals with short relative arm length. Yet, this has not been demonstrated in the experimental setting. ${ }^{4}$ One method of bringing the shoulders closer to the supporting surface and thereby increasing the functional arm length is to flex forward. Although the individual flexed further forward with the orthosis, the heights of the acromion processes were not significantly different. Therefore, there was no relative increase in the functional arm length of the subject between conditions. Nevertheless, moving the trunk forward also brings the centre of mass forward and this may improve the biomechanical advantage of the shoulder depressors (working with humeral attachments fixed) to lift the pelvis. Such a notion would support inferences made by Bergstrom and co-workers. 4

\section{Reaching}

The use of the orthosis increased the reaching capability of the individual with C5 quadriplegia.
However, this was primarily a mechanism of the altered trunk posture. Plotting the trajectories relative to the acromion process demonstrated that changes in the upper extremity extension made a minimal contribution to the improved performance. A significant functional impact on the upper extremity reach may be reflected in the increased lateral excursion of the COP during the reaching task. Hill ${ }^{7}$ illustrated the fact that two handed reaching is virtually impossible for individuals with poor trunk stability. The provision of the orthosis may lead to improved bilateral upper extremity function via a mechanism of increased core (trunk) stability and altered body posture.

\section{Transfers}

The prototype orthosis did not alter the rotational movement strategy of the individual in this trial. The subject demonstrated a very stable movement pattern without the orthosis. When using the orthosis the variability of the motor pattern analysis increased. From this, it would seem that the subject was still learning how to optimise the co-ordination of the head and trunk. Given that the subject had had a short familiarisation period it is clear that future research will endeavour to elucidate the efficacy of the orthosis over an extended practice period. It remains unclear if the orthosis, in increasing the core stability of the individual, provides any benefit for the individual to transfer the angular momentum from the head and shoulders to the pelvis when attempting to move the pelvis laterally.

Although there was a mean increase in the lateral displacement of the pelvis between phases it was not possible to differentiate this from the natural trend of the data. However, the fact that the subject was able to flex forward without using his arms for support may present a possible mechanism for a greater biomechanical optimisation of hand placements during transfers. This may lead to an improved overall performance by altering the lifting biomechanics. In any case, the clinical significance (as opposed to statistical significance) of the $3.6 \mathrm{~cm}$ increased lateral displacement of the pelvis when using the orthosis is yet to be elucidated.

Finally, the results of this study suggest that this prototype orthosis may have influenced the stability of the individual and altered the options for hand placements during the transfer. Significant development of the orthosis and a possible longterm training regimen may assist in understanding which mechanisms may influence the functional outcome of the individual. It may be possible that such a device may prove useful as a basis for future functional rehabilitation regimens which progress to a hybrid orthotic system.

\section{Conclusions}

The ability to transfer in individuals with tetraplegia represents a significant factor in their overall mobility. 
Most research to date, associated with SCI mobility, has been directed towards gait and is based on extensive and well reported fundamental biomechanical analysis of the gait cycle. The ability to transfer independently represents a greater functional improvment and is associated with the more dependent individuals when compared to walking in individuals with paraplegia. This study has utilised previous biomechanical research which has identified factors related to the ability to transfer, developed an orthosis on the basis of the biomechanical analysis, and demonstrated that the forward arm reach was increased in one individual with greater safe excursion of the COP during the dynamic phase of the reach. The improved arm reach was a result of increased forward flexion and a more extended lower trunk. Using the orthosis the subject was on average able to laterally displace the pelvis an additional $3.6 \mathrm{~cm}$ during an attempted transfer. This was found not to be statistically significant and the clinical significance is yet to be elucidated.

It is clear that further development of the system is required, yet it represents a possible unique solution, based on motion analysis principles, of transferring by dependent individuals with quadriplegia. The likely user population, the overall functional benefits, the compliance of the users and possible modifications towards an hybrid system are all possible directions for future research.

\section{Acknowledgements}

This study would not have been possible without the support of the Royal Perth Rehabilitation Hospital Bioengineering Department. The authors would like to thank Messrs B Scull, D Severn and P Klemp for their advice and assistance. The prototype orthosis has been previously presented ${ }^{12}$ at the International Functional
Electrical Stimulation Symposium held at Case Western Reserve University, Cleveland, Ohio, May 14-16, 1996.

\section{References}

1 Kralj AR, Bajd T. Functional electrical stimulation: standing and walking after spinal cord injury. Boca Raton: CRC Press Inc., 1989: p198.

2 Phillips CA. Functional Electrical Rehabilitation. New York: Springer-Verlag, 1991.

3 Yarkony GM et al. Rehabilitation outcomes in complete C5 quadriplegia. Archives of Physical Medicine and Rehabilitaiton 1988; 67(2): $73-76$.

4 Bergstrom EMK et al. Physical ability in relation to anthropometric measurements in persons with complete spinal cord lesion below the sixth cervical segment. International Rehabilitation Medicine 1985; 7: $51-55$.

5 Allison GT, Singer KP, Marshall RN. Transfer movement strategies of individuals with spinal cord injuries. Disability and Rehabilitation 1996; 18: $35-41$.

6 Allison GT, Singer KP, Marshall RN. Muscle activation patterns during transfers in individuals with spinal cord injury. Australian Journal of Physiotherapy 1995; 41: 3: 169-176.

7 Hill JP. Spinal Cord Injury. A guide to functional outcomes in occupational therapy. Rockville, Maryland: Aspen Publishers Inc., 1986: p248. Olson D, ed. The Rehabilitation Institute of Chicago Publication Series.

8 Frankel HL et al. The value of postural reduction in the initial management of closed injuries of the spine with paraplegia and tetraplegia. Part I. Paraplegia 1969; 7: 179-192.

9 Press WH, Flannery BP, Teukolsky SA, Vetterling WT. Numerical recipes: The art of scientific computing. [Fortran version]. Cambridge: Cambridge University Press, 1990: p702.

10 Stevens J. Applied multivariate statistics for the social sciences. (Second ed.) Hillsdale, New Jersey: Lawrence Erlbaum Associates, Publishers., 1992: p629.

11 Crosbie J, Sharpley CF. DMITSA A statistical program for analysing data from interrupted time-series. In: 2.0 ed. Victoria, Australia: Sharpley, C. Faculty of Education. Monash University, 1989.

12 Allison GT, Singer KP (1996). Electrically assisted transfers in quadriplegia. Proceedings of the First Annual Conference of the International FES Society, May 14-16, Case Western Reserve University, Cleveland, Ohio, USA. 